\title{
Improving gastric transit, gastrointestinal persistence and therapeutic efficacy of the probiotic strain Bifidobacterium breve UCC2003
}

\author{
Vivien M. Sheehan, ${ }^{1} \dagger$ Roy D. Sleator, ${ }^{2} \dagger$ Colin Hill ${ }^{1,2}$ and \\ Gerald F. Fitzgerald ${ }^{1,2}$
}

Correspondence

Roy D. Sleator

r.sleator@ucc.ie

Received 29 January 2007

Revised 26 May 2007

Accepted 22 June 2007

\author{
${ }^{1}$ Department of Microbiology, University College Cork, Ireland \\ ${ }^{2}$ Alimentary Pharmabiotic Centre, University College Cork, Ireland
}

\section{INTRODUCTION}

As population demographics shift towards a more elderly society (WHO, 2002), the incidence of functional gastrointestinal disorders is becoming more prevalent (Greenwald, 2004; Hébuterne, 2003; Pilotto, 2004). This, coupled with an increasing dependence on conventional antibiotics and the emergence of antibiotic-resistant bacteria, poses significant challenges to the effective treatment of gastrointestinal disorders. However, the potential prophylactic and therapeutic properties attributed to certain probiotic bacteria may offer an effective alternative (Sleator \& Hill, 2007a). Indeed, numerous clinical studies have attributed a myriad of impressive health-promoting effects to probiotics, including effective treatment of certain digestive and metabolic disorders (Leahy et al., 2005) as well as antagonistic activities against microbial pathogens (Asahara et al., 2001, 2004; Gagnon et al., 2006; Servin, 2004; Silva et al., 1999).

This study focuses on the probiotic strain Bifidobacterium breve UCC2003, the genome of which has recently been sequenced (S. C. Leahy and others, unpublished data). While studies concerning the clinical efficacy of strain UCC2003 are at a preliminary stage, recent studies have

†These authors contributed equally to this work. identified a number of potential health-promoting properties (Coakley et al., 2003), making it a potentially important probiotic culture. However, while genomic analysis has identified a number of stress adaptation systems employed by B. breve UCC2003 (Ventura et al., 2004a, 2005a, b, c, d, e, 2006), the genome appears devoid of obvious compatible solute-uptake systems. Found in numerous prokaryotic and eukaryotic species, compatible solute accumulation has been shown to protect a variety of cell types under a range of stressful conditions by maintaining cytoplasmic protein and membrane integrity (Abee \& Wouters, 1999; Sleator et al., 2000, 2001b, 2003a, b; Sleator \& Hill, 2002).

In order to survive and proliferate within the gastrointestinal tract, probiotics must tolerate several environmental hurdles, including the low $\mathrm{pH}$ of the stomach, as well as reduced water activity $\left(a_{\mathrm{w}}\right)$ and bile in the upper small intestine. Furthermore, the ability to persist in the intestine is considered to be a valuable criterion in achieving optimal probiotic efficacy (Vaughan et al., 2002). While many bifidobacteria have evolved to tolerate the stresses of gastrointestinal transit (Asahara et al., 2004; Fujiwara et al., 2001; Gagnon et al., 2006; Picard et al., 2005), significant variation exists among different strains. Thus, the application of processes to improve the physiological robustness of probiotic cultures in vivo is a clinical imperative (Sleator \& Hill, 2006, 2007b, c). 
Pathogenic species, with life cycles bridging the host and external milieu, have evolved strategies to ensure their continued survival in these diverse ecological niches (Hill et al., 2002; Sleator \& Hill, 2002, 2005). Thus, pathogenic genera represent a useful reservoir of stress survival mechanisms which could potentially improve the physiological robustness and clinical effectiveness of probiotic strains. The term 'patho-biotechnology', coined by Sleator \& Hill (2006), describes the exploitation of pathogenic stress-survival strategies in the design of more versatile probiotic cultures (Sleator \& Hill, 2006, 2007b, c).

Previously the in vitro stress tolerance of the probiotic strain Lactobacillus salivarius UCC118 was significantly improved by heterologous expression of the betaine uptake system BetL, isolated from Listeria monocytogenes (Sheehan et al., 2006). Herein, we describe a similar approach to improve the gastric transit, gastrointestinal persistence and therapeutic efficacy of Bifidobacterium breve UCC2003. This study thus provides the first in vivo experimental evidence in support of the patho-biotechnology concept.

\section{METHODS}

Bacterial strains, media and growth conditions. Bacterial strains and plasmids used in this study are listed in Table 1. Bifidobacterium breve UCC2003 (previously designated NCIM B8807), originally isolated from an infant nursing stool (Ventura et al., 2004b), was routinely cultured in reinforced clostridial medium (RCM) at $37{ }^{\circ} \mathrm{C}$ in an anaerobic chamber (Don Whitley Scientific). Where mentioned, a defined medium (DM) based on that described by Kongo et al. (2003) was used to culture B. breve, with glycine betaine (Sigma) being added as a filter-sterilized solution. Escherichia coli MKH13 was grown in Luria-Bertani (LB) medium at $37^{\circ} \mathrm{C}$ with agitation. $L$. monocytogenes was grown with aeration at $37{ }^{\circ} \mathrm{C}$ in brain heart infusion (BHI) broth. A concentrated stock of the antibiotic chloramphenicol $(\mathrm{Cm})$ was prepared (Maniatis et al., 1982) and added to media at a concentration of $4 \mu \mathrm{g} \mathrm{ml}^{-1}$ and $7.5 \mu \mathrm{g} \mathrm{ml}^{-1}$ when selecting for bifidobacteria and Listeria respectively. All media were supplied by Oxoid.
Genetic manipulations. Plasmid DNA isolation was performed using the Qiagen QIAprep spin miniprep kit. PCR was performed using a Hybaid PCR Express system. Oligonucleotide primers for PCR were synthesized by Sigma-Genosys Biotechnologies, and Taq DNA polymerase (Biotaq; Bioline) was used for all reactions. Colony PCR was carried out following lysis of cells at $96{ }^{\circ} \mathrm{C}$ for $10 \mathrm{~min}$ with IGEPAL CA-630 (Sigma). E. coli was transformed by standard methods (Maniatis et al., 1982) while electrotransformation of $B$. breve UCC2003 was performed as described by MacConaill et al. (2003). Essentially, mid-exponential-phase cells were chilled on ice for $20 \mathrm{~min}$ and this was followed by centrifugation. The cell pellet was washed twice and resuspended in $0.5 \mathrm{M}$ sucrose $/ 1 \mathrm{mM}$ citrate buffer ( $\mathrm{pH}$ 5.8). The cells were incubated on ice for $10 \mathrm{~min}$ followed by electrotransformation with a Bio-Rad Gene Pulser II apparatus under the following conditions: $25 \mu \mathrm{F}, 200 \Omega$ and $2.0 \mathrm{kV} \mathrm{cm}^{-1}$. RCM was added to the cells, and the mixture was incubated anaerobically at $37^{\circ} \mathrm{C}$ for $2.5 \mathrm{~h}$ prior to plating.

Plasmid construction. PCR primers with incorporated PstI ( $5^{\prime}$ CATCTGCAGGCTTTCTCCCCCTTTTTCCTC-3') and XbaI (5'CATTCTAGAGCTCTATTCCAATTACCGCCATTTC- $3^{\prime}$ ) restriction enzyme sites (underlined) were used to amplify the complete bet $L$ gene from the chromosome of $L$. monocytogenes LO28. The resultant PCR product was digested with PstI and XbaI and subsequently ligated into similarly digested pNZ8048 using T4 DNA ligase (Roche Diagnostics). The resultant plasmid, containing betL under the transcriptional control of its own promoter, was designated pNZ8048betL. Both pNZ8048betL and pNZ8048 (control) were initially introduced into E. coli MKH13, and plasmid DNA extracted from successful transformants was sequenced and subsequently transformed into B. breve UCC2003, giving rise to UCC2003$\mathrm{BetL}^{+}$. B. breve UCC2003 harbouring pNZ8048, used as a negative control, was designated UCC2003n.

Transcriptional analysis. $B$. breve UCC2003 cells were grown at $37{ }^{\circ} \mathrm{C}$ to mid-exponential phase in RCM. Cells were harvested by centrifugation and flash-frozen at $-80{ }^{\circ} \mathrm{C}$ with liquid nitrogen. Total RNA was extracted using the hot acid phenol procedure described by Ripio et al. (1998), and cDNA was synthesized by adding $1 \mu \mathrm{g}$ total RNA to $4 \mu \mathrm{l} 5 \times$ RT buffer (Roche), $2 \mu 1100 \mathrm{mM}$ dithiothreitol, $0.5 \mu \mathrm{l}$ of a deoxynucleoside triphosphate mix, $0.25 \mu \mathrm{l}$ RNasin, $100 \mathrm{ng}$ of the random primer $\mathrm{p}(\mathrm{dN})_{6}$, and $1 \mu \mathrm{l}$ Expand reverse transcriptase (Roche). The reaction mixture was incubated at $42{ }^{\circ} \mathrm{C}$ for $9 \mathrm{~h}$.

Table 1. Bacterial strains and the plasmids used in this study

\begin{tabular}{|c|c|c|}
\hline Strain or plasmid & Relevant properties ${ }^{\star}$ & Source or reference \\
\hline \multicolumn{3}{|l|}{ Strains } \\
\hline \multicolumn{3}{|l|}{ B. breve } \\
\hline UCC2003 & Wild-type parent strain & UCC culture collection \\
\hline UCC2003n & UCC2003 containing the cloning vector pNZ8048 & This study \\
\hline UCC2003-BetL ${ }^{+}$ & UCC2003 containing the cloning vector pNZ8048betL & This study \\
\hline \multicolumn{3}{|l|}{ E. coli } \\
\hline MKH13 & $\mathrm{MC} 4100 \Delta($ putPA $) 101 \Delta($ proP $) 2 \Delta($ proU $)$ & Kempf \& Bremer (1995) \\
\hline \multicolumn{3}{|l|}{ L. monocytogenes } \\
\hline $\mathrm{LO} 28$ & Serotype $1 / 2 c$ & P. Cossart, Institut Pasteur, Paris \\
\hline EGD-e & Sequenced strain & UCC culture collection \\
\hline \multicolumn{3}{|l|}{ Plasmids } \\
\hline pNZ8048 & $\mathrm{Cm}^{\mathrm{r}}$ & De Ruyter et al. (1996) \\
\hline pNZ8048betL & $\mathrm{Cm}^{\mathrm{r}}$, harbouring bet $\mathrm{L}$ under the control of the listerial promoter & This study \\
\hline
\end{tabular}

${ }^{*} \mathrm{Cm}^{\mathrm{r}}$, chloramphenicol resistant. 
In all cases, control PCR reactions were used to ensure the complete removal of DNA from RNA preparations prior to reverse transcription. Oligonucleotide primers betLF (5'-AATCGTGCTTCCCGCTTTAGTC- $\left.3^{\prime}\right)$ and betLR (5'-GCACACTTGCTATTCCCGCTTG-3') were used for PCR amplification of a 314 bp betL DNA fragment from UCC2003-BetL ${ }^{+}$. All glass and plastic-ware used in RNA analysis was first treated with $2 \%$ SDS for $15 \mathrm{~min}$, before rinsing with $1: 10$ in diethyl pyrocarbonate (DEPC)-treated water.

Resistance to gastric acid and low pH. Simulated gastric juice was prepared by suspending pepsin $(0.3 \% \mathrm{w} / \mathrm{v})$ (Sigma) in saline $(0.5 \%$, $\mathrm{w} / \mathrm{v}$ ). The solution, with or without $5 \mathrm{mM}$ added betaine (Sigma), was adjusted to $\mathrm{pH} 2.5$ with $\mathrm{HCl}$ and filter-sterilized through a $0.45 \mu \mathrm{M}$ membrane (Charteris et al., 1998). Also, RCM was adjusted to $\mathrm{pH} 2.5$ using $\mathrm{HCl}$. Overnight cultures of B. breve UCC2003-BetL $\mathrm{U}^{+}$ and UCC2003n were inoculated (3\%) into the simulated gastric juice and $\mathrm{pH}$-modified RCM. Both solutions were incubated anaerobically at $37{ }^{\circ} \mathrm{C}$. Viable cell counts were performed by diluting cultures in quarter-strength Ringer's solution and enumerating on reinforced clostridial agar (RCA).

Osmotolerance. Overnight cultures of B. breve UCC2003-BetL $\mathrm{U}^{+}$ and UCC2003n were washed twice in Ringer's solution before being inoculated $(2 \%)$ into DM and DM supplemented with $1.5 \%(\mathrm{w} / \mathrm{v})$ $\mathrm{NaCl}$ or $6 \%(\mathrm{w} / \mathrm{v})$ sucrose, both of which represent an osmolarity equivalent to that encountered in the upper small intestine (Gupta \& Chowdhury, 1997). Strains were grown statically at $37{ }^{\circ} \mathrm{C}$ and $\mathrm{OD}_{600}$ readings taken over $35 \mathrm{~h}$. Optical density values were confirmed by viable plate counts.

Bile tolerance. Human bile, obtained from the gallbladder of patients undergoing laparoscopic cholecystectomy, was filter-sterilized through a $0.45 \mu \mathrm{m}$ membrane before being added to cooled sterilized RCM. The concentration (1:10 dilution), pH 5.5 and osmolarity $(0.3 \mathrm{M} \mathrm{NaCl})$ of human bile were adjusted to mimic the environment of the upper small intestine, into which bile is secreted. Overnight cultures of $B$. breve UCC2003-BetL ${ }^{+}$and UCC2003n were inoculated $(3 \%)$ into the bile preparation. Samples were incubated anaerobically at $37{ }^{\circ} \mathrm{C}$ and viability monitored by diluting cultures in one-quarter-strength Ringer's solution and enumerating onto RCA.

\section{Survival of $B$. breve derivatives following exposure to suc- cessive stress treatments mimicking in vivo conditions. Overnight cultures of B. breve $\mathrm{UCC}^{2003-\mathrm{BetL}^{+}}$and UCC2003n were centrifuged, washed with PBS and resuspended in simulated gastric juice $\mathrm{pH} 2.5$ for $90 \mathrm{~min}$. Cells were then harvested by centrifugation and resuspended in human bile, adjusted to the osmolarity of the gut, for $90 \mathrm{~min}$. Experiments were performed in triplicate with and without $5 \mathrm{mM}$ added betaine. Viable cell counts were performed by diluting cultures in quarter-strength Ringer's solution and enumerat- ing on RCA.}

Survival of $B$. breve UCC2003 derivatives in the murine model. Overnight cultures of $B$. breve strains UCC2003-BetL ${ }^{+}$and UCC2003n were centrifuged, washed with PBS and used to inoculate 8-12-week-old female BALB/c mice, five per treatment group. Colonization of bifidobacteria was established by three consecutive daily administrations whereby each animal received $20 \mu \mathrm{l}$ of $\sim 5 \times 10^{9}$ cells using a micropipette tip placed immediately behind the incisors (Sleator et al., 2001a). In vivo survival of B. breve UCC2003 derivatives was monitored by periodic examinations of viable counts in faecal pellets. Briefly, fresh faecal pellets extracted by scuffing each animal were weighed, homogenized in quarter-strength Ringer's solution, diluted and plated onto RCA/Cm plates. Thirty-two days postinfection mice were euthanased and the bifidobacterial numbers in the intestines and caecum of infected animals were determined by spread-plating homogenates onto RCA/Cm plates. PCR primers specific to the $B$. breve $16 \mathrm{~S}$ rRNA region [bifF (5'-AGAAAGGAGGTGATCCAGCCGCAC- $3^{\prime}$ ) and bifR (5'-TTTTTGTGGAGGGTTCGATTCTGGCTCAGGATGA-3'); S. C. Leahy and others, unpublished data] were used to confirm that $\mathrm{Cm}^{\mathrm{r}}$ bifidobacteria isolated from the faecal pellets were indeed $B$. breve, while PCR primers specific to bet $L$ (betLF and betLR) confirmed the presence of the gene in UCC2003BetL $^{+}$. Furthermore, faecal pellets taken before administration with bifidobacteria showed no background microflora when plated on the antibiotic selection plates.

Virulence assays. Colonization of $B$. breve UCC2003 derivatives was established by three successive daily oral administrations as described above. Mice in the control group received PBS. On the fourth day, $L$. monocytogenes EGD-e was pelleted by centrifugation, washed with PBS, and used to orally inoculate the animals with $\sim 10^{11}$ c.f.u. $\mathrm{ml}^{-1}$. On the same day, faecal samples were taken from each animal and the numbers of recoverable UCC2003-BetL ${ }^{+}$and UCC2003n were assessed. PCR was used to confirm that $\mathrm{Cm}^{\mathrm{r}}$ bifidobacteria isolated from the faecal pellets were $B$. breve UCC2003. Three days postinfection animals were sacrificed, individual livers and spleens were homogenized in PBS, and serial dilutions were plated onto BHI/Cm agar plates.

Statistical analysis. Treatment comparisons were performed using ANOVA test at $95 \%$ significance, using the Systat software version 9 (SPSS).

\section{RESULTS}

\section{bet $L$ expression analysis}

RT-PCR analysis gave a single 314 bp band for UCC2003$\mathrm{BetL}^{+}$, indicating active transcription of the betL gene in the complemented strain (Fig. 1). As expected, no band was observed for the control strain UCC2003n lacking betL.

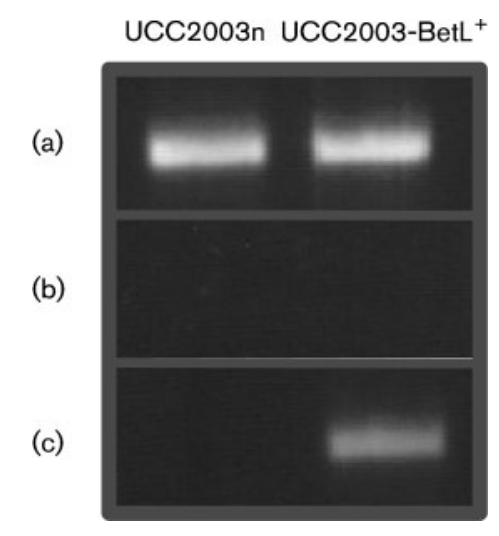

Fig. 1. RT-PCR analysis of betL expression in UCC2003-BetL ${ }^{+}$ (a) Control PCRs using cDNA template and 16S rRNA-specific primers bifF and bifR confirm that equal levels of RNA were extracted for UCC2003n and UCC2003-BetL ${ }^{+}$. (b) No products were obtained when RNA was used as template with bifF and bifR, confirming no DNA carryover. (c) betL-specific primers betLF and betLR amplify a 314 bp fragment from UCC2003-BetL ${ }^{+}$cDNA, while as expected no product was obtained when the cDNA of the control strain UCC2003n was used as template. All bands are the result of 22 PCR cycles. 
Control PCRs on total cDNA from both UCC2003-BetL ${ }^{+}$ and UCC2003n using the 16S rRNA bifF and bifR primers gave bands of equal intensity, confirming that equal levels of RNA were extracted for the control and complemented strains. However, when total RNA was used as a template no product was obtained, confirming the absence of any chromosomal DNA.

\section{Tolerance of $B$. breve UCC2003 to gastric acid is significantly improved by the addition of betL}

Survival of B. breve UCC2003-BetL ${ }^{+}$and UCC2003n was monitored following exposure to simulated gastric juice adjusted to $\mathrm{pH} 2.5$ in the presence and absence of $5 \mathrm{mM}$ added betaine (Fig. 2). UCC2003-BetL ${ }^{+}$was recovered at significantly $(P<0.05)$ higher levels than UCC2003n, 2 and $3 \mathrm{~h}$ post-exposure in the presence of betaine. No significant differences were detected in the absence of betaine. Neither strain (UCC2003-BetL ${ }^{+}$or UCC2003n) was detected following $30 \mathrm{~min}$ exposure to RCM adjusted to $\mathrm{pH} 2.5$ (data not shown).

\section{BetL enhances the osmotolerance of $B$. breve UCC2003}

Betaine accumulation has long been associated with an increase in osmotolerance. Initially, growth of UCC2003$\mathrm{BetL}^{+}$and UCC2003n in DM at $37{ }^{\circ} \mathrm{C}$ was compared to assess whether the presence of betL had an effect on the growth rate in the absence of additional $\mathrm{NaCl}$ (Fig. 3a). No difference in the growth rate or the extent of growth was observed, suggesting that the presence of betL has no impact under normal, non-stress growth conditions. However, with $6 \%$ added sucrose (Fig. 3b), UCC2003$\mathrm{BetL}^{+}$displayed a faster growth rate and reached a higher final optical density than UCC2003n. Similarly, when both strains were exposed to DM supplemented with $1.5 \% \mathrm{NaCl}$

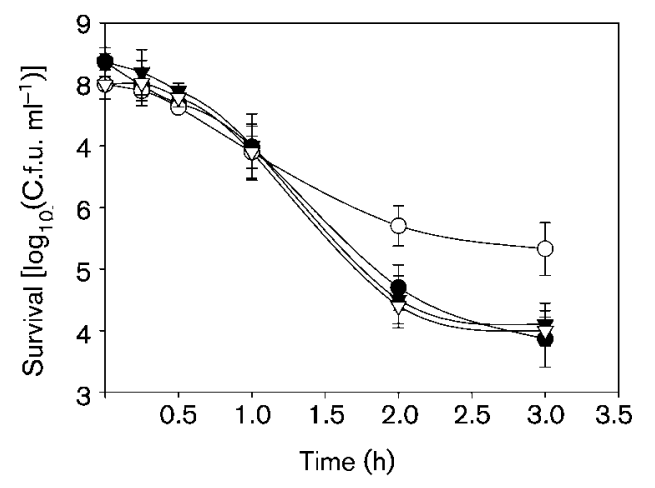

Fig. 2. Survival of $B$. breve UCC2003-BetL ${ }^{+}$(circles) and

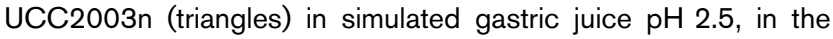
presence (open symbols) and absence (closed symbols) of $5 \mathrm{mM}$ added betaine. Standard error of triplicate experiments was determined for each data point.
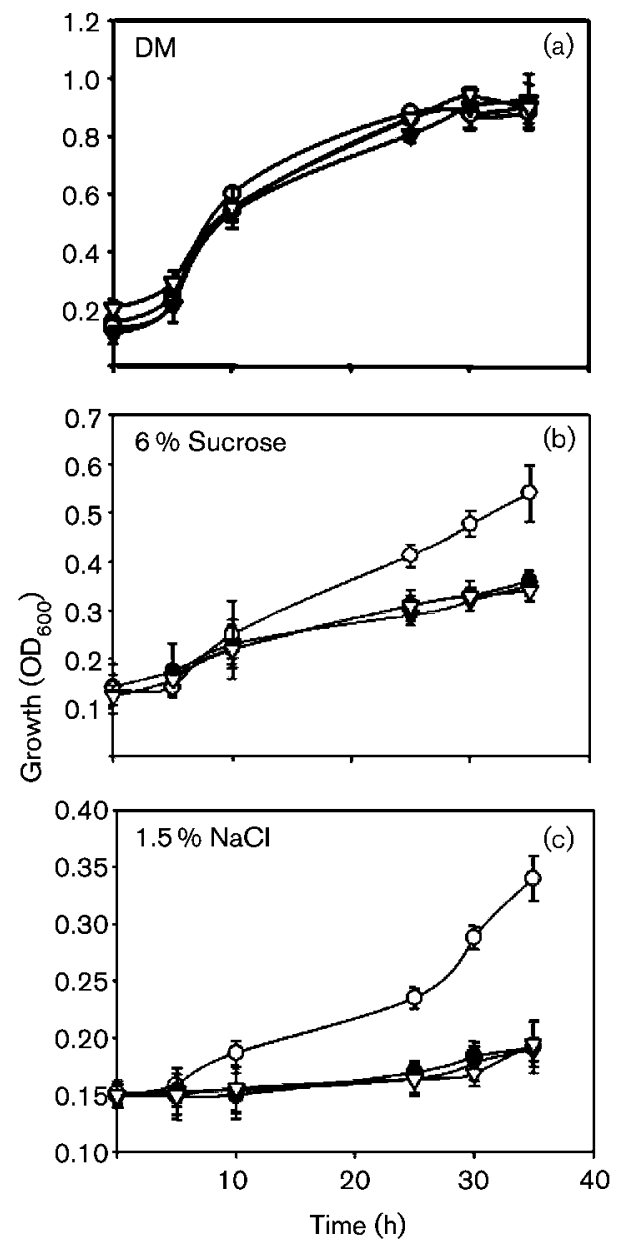

Fig. 3. Growth of B. breve UCC2003-BetL ${ }^{+}$(circles) and UCC2003n (triangles) in DM (a) at $37{ }^{\circ} \mathrm{C}$ under normal physiological conditions, with $6 \%(\mathrm{w} / \mathrm{v})$ added sucrose (b) and with $1.5 \%(\mathrm{w} / \mathrm{v})$ added $\mathrm{NaCl}(\mathrm{c})$ in the presence (open symbols) and absence (filled symbols) of $5 \mathrm{mM}$ added betaine. Both $1.5 \%$ $\mathrm{NaCl}$ and $6 \%$ sucrose represent an osmolarity equivalent to that encountered in the upper small intestine. Cell growth was measured spectrophotometrically by determining $\mathrm{OD}_{600}$. Each point represents the mean \pm SEM of at least three independent experiments.

(Fig. 3c), UCC2003-BetL ${ }^{+}$again displayed a significantly faster growth rate than UCC2003n and also reached a high final optical density.

\section{The presence of betL in B. breve UCC2003 does not provide protection against bile}

When cultures were challenged with human bile, betL was unable to enhance the tolerance of B. breve UCC2003 to the biological detergent beyond that achieved by the control strain, UCC2003n. Both UCC2003-BetL ${ }^{+}$and UCC2003n displayed similar levels of resistance to human bile, showing a $1.5 \log$ reduction in c.f.u. after $6 \mathrm{~h}$ exposure (data not shown). 


\section{B. breve UCC2003-BetL ${ }^{+}$is recovered at higher levels than UCC2003n following successive stress treatments mimicking in vivo conditions}

$\mathrm{UCC} 003-\mathrm{BetL}^{+}$was recovered at higher levels than UCC2003n after exposure to a simulated gastric juice followed immediately by exposure to human bile adjusted to mimic conditions encountered in vivo (Fig. 4). The only significant differences were observed following exposure to the successive stress treatments in the presence of $5 \mathrm{mM}$ added betaine, UCC2003-BetL ${ }^{+}$remaining viable at significantly $(P<0.05)$ higher levels than the control strain UCC2003n. The slightly higher survival levels observed for UCC2003-BetL ${ }^{+}$in the absence of added betaine most likely result from betaine pre-accumulation during inoculum preparation.

\section{Recovery of $B$. breve UCC2003 derivatives from faecal samples, intestines and caecum of BALB/C mice}

Following oral inoculation of $\mathrm{BALB} / \mathrm{c}$ mice with $B$. breve UCC2003-BetL $^{+}$or UCC2003n for three consecutive days, both strains could be recovered from the murine intestine. A population greater than $10^{6}$ c.f.u. $\mathrm{g}^{-1}$ was recorded in the faecal contents for up to 32 days (Fig. 5a). Viable counts of UCC2003-BetL ${ }^{+}$were significantly $(P<0.05)$ greater than those of UCC2003n during the initial 3 days of

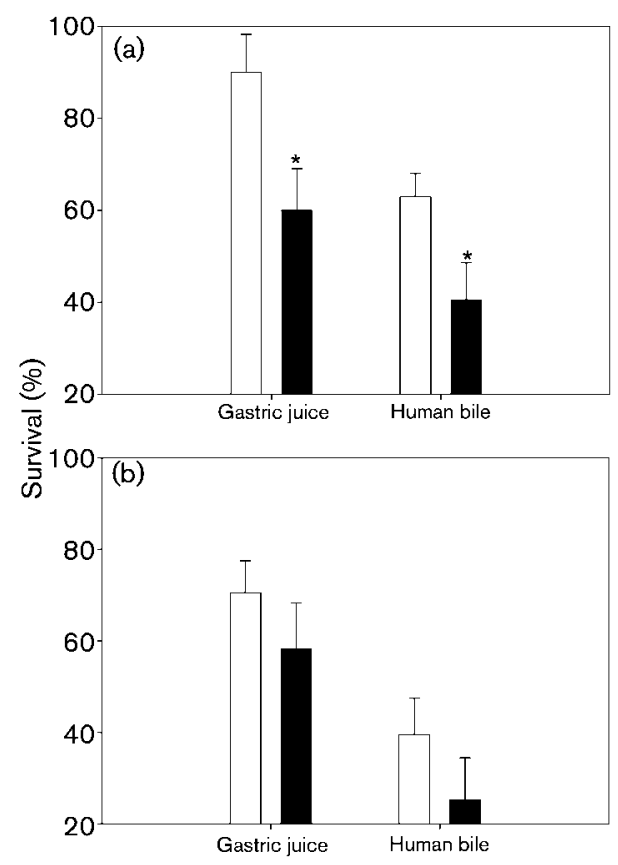

Fig. 4. Survival of $B$. breve UCC2003-BetL ${ }^{+}$(white bars) and UCC2003n (black bars) (a) with and (b) without $5 \mathrm{mM}$ added betaine in simulated gastric juice for 90 min, mimicking conditions encountered in the stomach, followed by exposure to human bile for $90 \mathrm{~min}$, representing conditions encountered in the upper small intestine. Asterisks represent significant differences. monitoring post-feeding. UCC2003-BetL ${ }^{+}$and UCC2003n were recovered at similarly high levels between days 6 and 10 , after which time a significant $(P<0.001)$ decrease in UCC2003n numbers was noted between days 10 and 14 . Colonization by B. breve UCC2003n stabilized on day 14 , with no significant decline in cell numbers recorded from then until the completion of the trial. However, while counts of UCC2003-BetL ${ }^{+}$also stabilized at day 14, this strain was recovered in numbers significantly $(P<0.05)$ greater than UCC2003n from day 14 onwards. On day 32, mice were sacrificed and the levels of both strains in the small and large intestines and caecum were assessed (Fig. 5b). UCC2003-BetL ${ }^{+}$was recovered at significantly $(P<0.01)$ higher levels in the large intestine and caecum when compared to UCC2003n.

\section{Listerial infection in the organs of mice is reduced following consumption of B. breve UCC2003 harbouring betL}

Consistent with previous observations, higher levels of UCC2003-BetL ${ }^{+}$were recovered from the faecal pellets following oral inoculation, suggesting that BetL improves the gastrointestinal persistence of the probiotic (data not shown). In order to ascertain whether the higher colonization rates would enhance or compromise the therapeutic benefits of the strain, the extent of listerial infection in the livers (Fig. 6a) and spleens (Fig. 6b) of mice fed UCC2003-BetL ${ }^{+}$, UCC2003n and PBS were compared. While the numbers of organ-specific c.f.u. recovered from the livers did not differ significantly, listerial proliferation in the spleen was significantly $(P<0.05)$ lower in mice fed B. breve UCC2003-BetL ${ }^{+}$in comparison to the two control groups. It is therefore reasonable to conclude that $B$. breve UCC2003-BetL $^{+}$demonstrates enhanced clinical efficacy when challenged with L. monocytogenes.

\section{DISCUSSION}

In order for bifidobacteria to exert a probiotic effect, it is essential that they remain viable within the delivery vehicle (food or tablet formulation), survive passage through the gastrointestinal tract and reach the intestine in sufficiently high numbers (Dunne et al., 2001; Tuomola et al., 2001). Previously we described the successful expression of bet $L$ in Lb. salivarius UCC118 (Sheehan et al., 2006) and in E. coli (Sleator et al., 1999, 2003c), demonstrating significantly improved survival of both strains to stresses encountered in foods and food-processing environments. In the present study we report the potential of the approach to enhance the gastrointestinal persistence and clinical efficacy of $B$. breve, a clinically important probiotic culture.

Survival of $B$. breve was significantly enhanced by the presence of betL when exposed to a simulated gastric juice $\mathrm{pH}$ 2.5. This is the first evidence that bet $L$ may play a role in improving probiotic viability during transit through the stomach. Interestingly, when cultures were exposed to a 

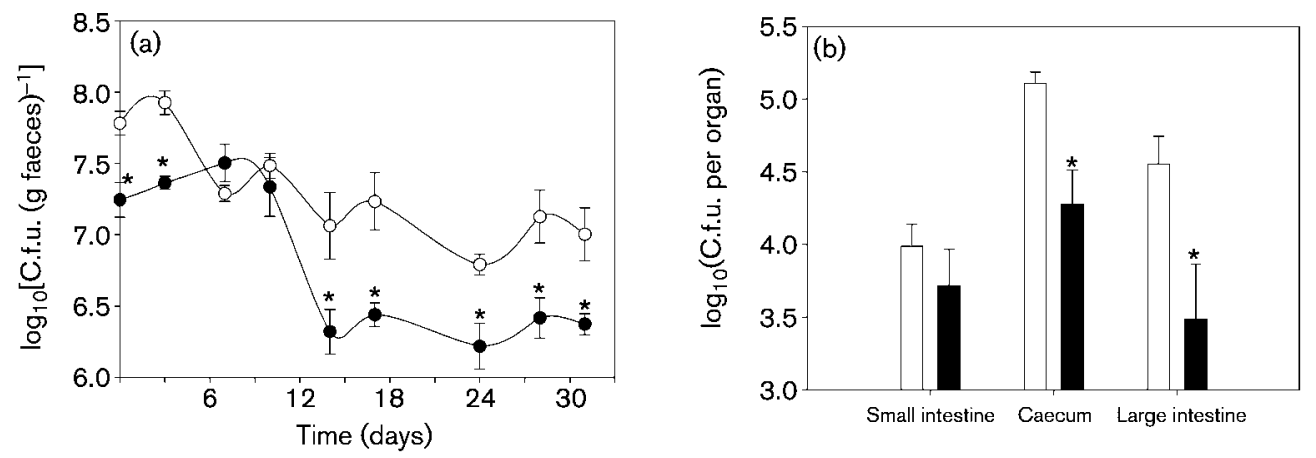

Fig. 5. Recovery of B. breve UCC2003-BetL ${ }^{+}(\bigcirc)$ and UCC2003n (๑) from female BALB/c mice over 32 days of analysis. (a) Faeces for bacteriological analysis were obtained from five mice in each treatment group and viable counts of $B$. breve UCC2003 derivatives were determined as described in the text. (b) Recovery of B. breve UCC2003-BetL ${ }^{+}$(white bars) and UCC2003n (black bars) from the small and large intestine and the caecum of the murine model after 32 days. The error bars represent the standard error of five animals $(n=5)$. Asterisks represent significant differences.

complex medium (RCM) adjusted to a similar $\mathrm{pH}$ (using $\mathrm{HCl}$ ), no significant difference in the acid tolerance of the strains was observed, implying that $\mathrm{pH}$ alone is not responsible for the improved viability of UCC2003-BetL ${ }^{+}$ in simulated gastric juice. Furthermore, UCC2003 derivatives declined at a significantly faster rate in the complex broth compared to the simulated gastric juice. Previously, Saarela et al. (2005) observed that pepsin protects bifidobacteria at $\mathrm{pH} 2.5$, suggesting that the reduced toxicity associated with the simulated gastric juice, as opposed to RCM, might be attributed to the presence of pepsin. In any case, improved survival of the complemented strain in gastric juice containing added betaine suggests that it is the compatible solute (accumulated via BetL) that is responsible for the observed increased tolerance to the gastric juice. Interestingly, in support of this observation Termont et al. (2006) recently reported improved tolerance to gastric juice in a Lactococcus lactis strain expressing trehalose-synthesizing genes from $E$. coli, suggesting a protective role for compatible solutes in the gastric environment.
Once in the upper small intestine, probiotics are exposed to low $a_{\mathrm{w}}$ (equivalent to $0.3 \mathrm{M} \mathrm{NaCl}$ ) (Gupta \& Chowdhury, 1997) and bile salts (at a concentration of $5 \mathrm{mM}$ ) (De Boever \& Verstraete, 1999). Consistent with our previous observations with $L b$. salivarius UCC118 (Sheehan et al., 2006), a significant osmoprotective effect was observed following the introduction of betL into $B$. breve, facilitating growth of the probiotic in conditions similar to those encountered in vivo $(1.5 \% \mathrm{NaCl}$ and $6 \%$ sucrose, both of which approximate the osmolarity of the gut). The presence of bet $L$ increased the growth rate of the probiotic and enabled the transformed strain to reach a higher final optical density compared to the control. As well as elevated osmolarity, the biological detergent bile also poses a significant hurdle to microbes in the upper small intestine (Sleator et al., 2005; Sleator \& Hill, 2007d). However, betL appeared to offer no protection to $B$. breve challenged with human bile, a result not altogether surprising given that BetL, or indeed betaine, has not previously been linked to bile tolerance. Finally, combining the gut-associated stresses in a sequence mimicking that encountered in vivo
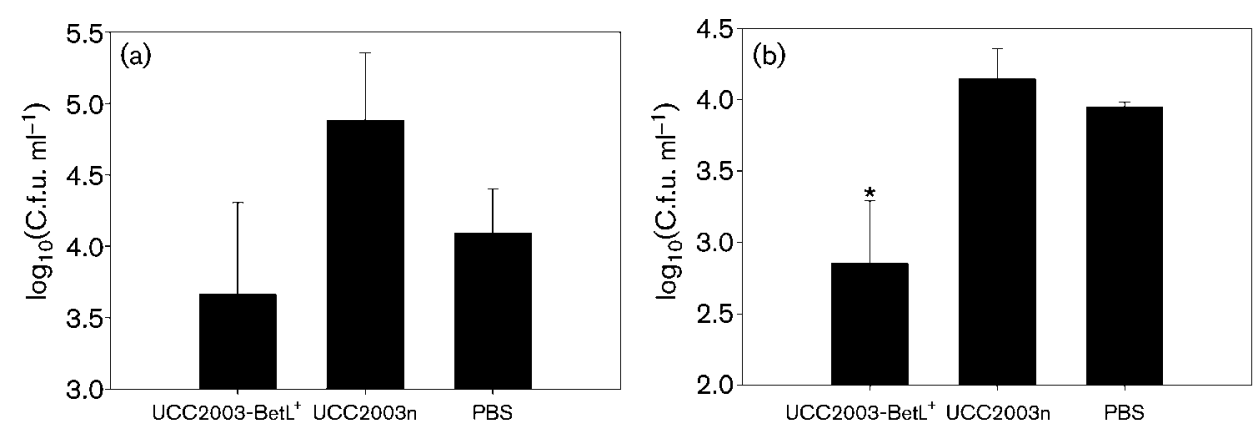

Fig. 6. Listerial infection in the livers (a) and spleens (b) of BALB/c mice. Animals were fed $\sim 10^{9}$ c.f.u. $\mathrm{ml}^{-1}$ of either UCC2003-BetL ${ }^{+}$or UCC2003n for three consecutive days. The control group was fed PBS. On the fourth day, all animals were infected with $\sim 10^{11}$ c.f.u. $\mathrm{ml}^{-1}$ L. monocytogenes EGD-e. Three days post-listerial infection the animals were sacrificed and the numbers of listeria were determined. Asterisks represent significant differences. 
confirmed that UCC2003-BetL ${ }^{+}$survived significantly better than the control strain in conditions similar to those encountered during gastric transit.

Following in vitro studies, in vivo analysis using $\mathrm{BALB} / \mathrm{C}$ mice demonstrated stable intestinal colonization of $B$. breve UCC3003 derivatives following oral inoculation. A possible explanation for the initial differences observed in the faecal pellets (days 1-3) may be greater numbers of UCC3003BetL $^{+}$isolates surviving gastric transit, a consequence of the greater resistance to gastric fluid. Once clear of the stomach, the elevated osmolarity of the intestinal tract likely promotes increased growth and survival of the BetL-complemented strain relative to the control. These findings correlate with previous observations by Sleator et al. (2001a) in which another compatible solute-uptake system (OpuC; facilitating both carnitine and betaine uptake) in L. monocytogenes was identified as an essential element for optimal intestinal survival of Listeria. Significantly higher levels of UCC2003$\mathrm{BetL}^{+}$were recovered from the colon, the recognized environmental niche for bifidobacteria. However, no significant differences in recoverable $B$. breve UCC2003 derivatives were observed in the small intestine, a finding that most likely reflects the fact that the small intestine is rich in bile, against which we have shown BetL offers no protective effect over and above that of the wild-type.

It seems reasonable to correlate increased delivery of probiotics to the intestine with improved therapeutic function, but this remained to be confirmed in vivo. A number of studies have reported that intestinal infections in the murine model can be prevented or alleviated by the administration of bifidobacteria that alter the microecology of the gut (Asahara et al., 2001, 2004; Gagnon et al., 2006). Asahara et al. (2001) demonstrated that antibiotic-induced proliferation and translocation of Salmonella enterica serovar Typhimurium into the host is markedly inhibited by precolonization of the intestine by $B$. breve strain Yakult. Furthermore, feeding $B$. breve strain Yakult to mice with a disrupted microbiota resulted in a large number of probiotics colonizing the intestine for a period of at least 2 weeks (Asahara et al., 2004). Moreover, infection with Shiga-toxin-producing E. coli O157:H7 was dramatically inhibited. Both studies suggested that the $\mathrm{pH}$-lowering effects of the probiotic appeared to be important for enabling these anti-infectious activities. Furthermore, $B$. breve strains have been reported to display antimicrobial activity against pathogens through competitive exclusion (Lievin et al., 2000), whereby probiotic strains with similar adherence mechanisms inhibit the attachment and colonization of the pathogen at these binding sites, thus protecting the host from infection (Marco et al., 2006).

In the present study, we report for the first time that feeding $B$. breve UCC2003-BetL ${ }^{+}$prior to listerial administration can protect the animal from infection. While mice administered UCC2003n showed no obvious decline in listerial numbers relative to those administered PBS, $B$. breve UCC2003 complemented with betL had a significant effect on listerial infection, particularly in the spleen. This is significant, given that splenic infection is an indicator of total systemic infection. Furthermore, damage to the spleen further weakens the body's immune response, increasing the mortality rate in severe infections. To our knowledge, this is the first clear evidence of an enhanced therapeutic effect following precise bio-engineering of a probiotic strain.

While we recognize that the introduction of genes from pathogens into probiotic cultures is unlikely to meet with immediate approval from regulatory authorities, this study proves that the concept of using such an approach to design more versatile cultures is at least scientifically valid. Following proof of concept, it is entirely likely that BetL homologues from GRAS (generally regarded as safe) organisms might offer similar protective effects. Furthermore, natural selection of probiotic cultures with elevated expression of such homologues might ultimately dispense with the necessity for recombinant DNA approaches altogether.

\section{ACKNOWLEDGEMENTS}

Dr Roy Sleator is a Health Research Board (HRB) Principal Investigator. The authors wish to acknowledge the continued financial assistance of the HRB and the Alimentary Pharmabiotic Centre (APC).

\section{REFERENCES}

Abee, T. \& Wouters, J. A. (1999). Microbial stress response in minimal processing. Int J Food Microbiol 50, 65-91.

Asahara, T., Nomoto, K., Shimizu, K., Watanuki, M. \& Tanaka, R. (2001). Increased resistance of mice to Salmonella enterica serovar Typhimurium infection by synbiotic administration of bifidobacteria and transgalactosylated oligosaccharides. J Appl Microbiol 91, 985-996.

Asahara, T., Shimizu, K., Nomoto, K., Hamabata, T., Ozawa, A. \& Takeda, Y. (2004). Probiotic bifidobacteria protect mice from lethal infection with shiga toxin-producing Escherichia coli O157: H7. Infect Immun 72, 2240-2247.

Charteris, W. P., Kelly, P. M., Morelli, L. \& Collins, J. K. (1998). Development and application of an in vitro methodology to determine the transit tolerance of potentially probiotic Lactobacillus and Bifidobacterium species in the upper human gastrointestinal tract. J Appl Microbiol 84, 759-768.

Coakley, M., Ross, R. P., Nordgren, M., Fitzgerald, G., Devery, R. \& Stanton, C. (2003). Conjugated linoleic acid biosynthesis by humanderived Bifidobacterium species. J Appl Microbiol 94, 138-145.

De Boever, P. \& Verstraete, W. (1999). Bile salt deconjugation by Lactobacillus plantarum 80 and its implications for bacterial toxicity. J Appl Microbiol 87, 345-352.

De Ruyter, P. G., Kuipers, O. P. \& de Vos, W. M. (1996). Controlled gene expression systems for Lactococcus lactis with the food-grade inducer nisin. Appl Environ Microbiol 62, 3662-3667.

Dunne, C., O'Mahony, L., Murphy, L., Thorton, G., Morrissey, D., O'Halloran, S., Feeney, M., Flynn, S., Fitzgerald, G. \& other authors (2001). In vitro selection criteria for probiotic bacteria of human origin: correlation with in vivo findings. Am J Clin Nutr 73, 386S-392S. 
Fujiwara, S., Seto, Y., Kimura, A. \& Hashiba, H. (2001). Intestinal transit of an orally administered streptomycin-rifampicin-resistant variant of Bifidobacterium longum SBT2928: its long-term survival and effect on the intestinal microflora and metabolism. J Appl Microbiol 90, 43-52.

Gagnon, M., Kheadr, E. E., Dabour, N., Richard, D. \& Fliss, I. (2006). Effect of Bifidobacterium thermacidophilum probiotic feeding on enterohemorrhagic Escherichia coli O157:H7 infection in BALB/c mice. Int J Food Microbiol 111, 26-33.

Greenwald, D. A. (2004). Aging, the gastrointestinal tract, and risk of acid-related disease. Am J Med 117 (Suppl 5A), 8S-13S.

Gupta, S. \& Chowdhury, R. (1997). Bile affects production of virulence factors and motility of Vibrio cholerae. Infect Immun 65, 1131-1134.

Hébuterne, X. (2003). Gut changes attributed to ageing: effects on intestinal microflora. Curr Opin Clin Nutr Metab Care 6, 49-54.

Hill, C., Cotter, P. D., Sleator, R. D. \& Gahan, C. G. M. (2002). Bacterial stress response in Listeria monocytogenes: jumping the hurdles imposed by minimal processing. Int Dairy J 12, 273-283.

Kempf, B. \& Bremer, E. (1995). OpuA, an osmotically regulated binding protein-dependent transport system for the osmoprotectant glycine betaine in Bacillus subtilis. J Biol Chem 270, 16701-16713.

Kongo, J. M., Gomes, A. M. P. \& Malcata, F. X. (2003). Development of a chemically defined medium for growth of Bifidobacterium animalis. J Food Sci 68, 2742-2746.

Leahy, S. C., Higgins, D. G., Fitzgerald, G. F. \& van Sinderen, D. (2005). Getting better with bifidobacteria. J Appl Microbiol 98, 1303-1315.

Lievin, V., Peiffer, I., Hudault, S., Rochat, F., Brassart, D., Neeser, J. R. \& Servin, A. L. (2000). Bifidobacterium strains from resident infant human gastrointestinal microflora exert antimicrobial activity. Gut 47, 646-652.

MacConaill, L. E., Fitzgerald, G. F. \& van Sinderen, D. (2003). Investigation of protein export in Bifidobacterium breve UCC2003. Appl Environ Microbiol 69, 6994-7001.

Maniatis, T., Fritsch, E. F. \& Sambrook, J. (1982). Molecular Cloning: a Laboratory Manual. Cold Spring Harbor, NY: Cold Spring Harbor Laboratory.

Marco, M. L., Pavan, S. \& Kleerebezem, M. (2006). Towards understanding molecular modes of probiotic action. Curr Opin Biotechnol 17, 204-210.

Picard, C., Fioramonti, J., Francois, A., Robinson, T., Neant, F. \& Matuchansky, C. (2005). Review article: bifidobacteria as probiotic agents - physiological effects and clinical benefits. Aliment Pharmacol Ther 22, 495-512.

Pilotto, A. (2004). Aging and upper gastrointestinal disorders. Best Pract Res Clin Gastroenterol 18, 73-81.

Ripio, M.-T., Vázquez-Boland, J.-A., Vega, Y., Nair, S. \& Berche, P. (1998). Evidence for expressional crosstalk between the central virulence regulator PrfA and the stress response mediator $\mathrm{ClpC}$ in Listeria monocytogenes. FEMS Microbiol Lett 158, 45-50.

Saarela, M., Virkajärvi, I., Alakomi, H.-L., Mattila-Sandholm, T., Vaari, A., Suomalainen, T. \& Mättö, J. (2005). Influence of fermentation time, cryoprotectant and neutralization of cell concentrate on freeze-drying survival, storage stability and acid and bile exposure of Bifidobacterium animalis ssp. lactis cells produced without milk-based ingredient. J Appl Microbiol 99, 1330-1339.

Servin, A. L. (2004). Antagonistic activities of lactobacilli and bifidobacteria against microbial pathogens. FEMS Microbiol Rev 28, 405-440.

Sheehan, V. M., Sleator, R. D., Fitzgerald, G. F. \& Hill, C. (2006). Heterologous expression of BetL, a betaine uptake system, enhances the stress tolerance of Lactobacillus salivarius UCC118. Appl Environ Microbiol 72, 2170-2177.

Silva, A. M., Bambirra, E. A., Oliveira, A. L., Souza, P. P., Gomes, D. A., Vieira, E. C. \& Nicoli, J. R. (1999). Protective effect of bifidus milk on the experimental infection with Salmonella enteritidis subsp. typhimurium in conventional and gnotobiotic mice. J Appl Microbiol 86, 331-336.

Sleator, R. D. \& Hill, C. (2002). Bacterial osmoadaptation: the role of osmolytes in bacterial stress and virulence. FEMS Microbiol Rev 26, 49-71.

Sleator, R. D. \& Hill, C. (2005). A novel role for the LisRK twocomponent regulatory system in listerial osmotolerance. Clin Microbiol Infect 11, 599-601.

Sleator, R. D. \& Hill, C. (2006). Patho-biotechnology; using bad bugs to do good things. Curr Opin Biotechnol 17, 211-226.

Sleator, R. D. \& Hill, C. (2007a). Probiotics as therapeutics for the developing world. J Infect Develop Countries (in press).

Sleator, R. D. \& Hill, C. (2007b). Patho-biotechnology; using bad bugs to make good bugs better. Sci Prog 90, 1-14.

Sleator, R. D. \& Hill, C. (2007c). 'Bioengineered bugs' - a pathobiotechnology approach to probiotic research and applications. Med Hypotheses, doi:10.1016/j.mehy.2007.03.008.

Sleator, R. D. \& Hill, C. (2007d). Gut osmolarity: a key environmental cue initiating the gastrointestinal phase of Listeria monocytogenes infection? Med Hypotheses, doi:10.1016/j.mehy.2007.02.028.

Sleator, R. D., Gahan, C. G. M., Abee, T. \& Hill, C. (1999). Identification and disruption of BetL, a secondary glycine betaine transport system linked to the salt tolerance of Listeria monocytogenes LO28. Appl Environ Microbiol 65, 2078-2083.

Sleator, R. D., Gahan, C. G. M., O'Driscoll, B. \& Hill, C. (2000). Analysis of the role of betL in contributing to the growth and survival of Listeria monocytogenes LO28. Int J Food Microbiol 60, 261-268.

Sleator, R. D., Wouters, J., Gahan, C. G. M., Abee, T. \& Hill, C. (2001a). Analysis of the role of OpuC, an osmolyte transport system, in salt tolerance and virulence potential of Listeria monocytogenes. Appl Environ Microbiol 67, 2692-2698.

Sleator, R. D., Gahan, C. G. M. \& Hill, C. (2001b). Mutations in the listerial $p r o B$ gene leading to proline overproduction: effects on salt tolerance and murine infection. Appl Environ Microbiol 67, 4560-4565.

Sleator, R. D., Francis, G. A., O’Beirne, D., Gahan, C. G. M. \& Hill, C. (2003a). Betaine and carnitine uptake systems in Listeria monocytogenes affect growth and survival in foods and during infection. J Appl Microbiol 95, 839-846.

Sleator, R. D., Gahan, C. G. M. \& Hill, C. (2003b). A postgenomic appraisal of osmotolerance in Listeria monocytogenes. Appl Environ Microbiol 69, 1-9.

Sleator, R. D., Wood, J. M. \& Hill, C. (2003c). Transcriptional regulation and posttranslational activity of the betaine transporter BetL in Listeria monocytogenes are controlled by environmental salinity. J Bacteriol 185, 7140-7144.

Sleator, R. D., Wemekamp-Kamphuis, H. H., Gahan, C. G. M., Abee, T. \& Hill, C. (2005). A PrfA-regulated bile exclusion system (BilE) is a novel virulence factor in Listeria monocytogenes. Mol Microbiol 55, 1183-1195.

Termont, S., Vandenbrouke, K., Iserentant, D., Neirynck, S., Steidler, L., Remaut, E. \& Rottiers, P. (2006). Intracellular accumulation of trehalose protects Lactococcus lactis from freeze-drying damage and bile toxicity and increases gastric acid resistance. Appl Environ Microbiol 72, 7694-7700.

Tuomola, E., Crittenden, R., Playne, M., Isolauri, E. \& Salminen, S. (2001). Quality assurance criteria for probiotic bacteria. Am J Clin Nutr 73, 393S-398S. 
Vaughan, E. E., de Vries, M. C., Zoetendal, E. G., Ben-Amor, K., Akkermans, A. D. \& de Vos, W. M. (2002). The intestinal LABs. Antonie Van Leeuwenhoek 82, 341-352.

Ventura, M., Canchaya, C., Zink, R., Fitzgerald, G. F. \& van Sinderen, D. (2004a). Characterization of the groEL and groES loci in Bifidobacterium breve UCC 2003: genetic, transcriptional and phylogenetic analyses. Appl Environ Microbiol 70, 6197-6209.

Ventura, M., van Sinderen, D., Fitzgerald, G. F. \& Zink, R. (2004b). Insights into the taxonomy, genetics and physiology of bifidobacteria. Antonie Van Leeuwenhoek 86, 205-223.

Ventura, M., Canchaya, C., Bernini, V., Del Casale, A., Dellaglio, F., Neviani, E., Fitzgerald, G. F. \& van Sinderen, D. (2005a). Genetic characterization of the Bifidobacterium breve UCC $2003 \mathrm{hrcA}$ locus. Appl Environ Microbiol 71, 8998-9007.

Ventura, M., Fitzgerald, G. F. \& van Sinderen, D. (2005b). Genetic and transcriptional organization of the clpC locus in Bifidobacterium breve UCC 2003. Appl Environ Microbiol 71, 6282-6291.

Ventura, M., Kenny, J. G., Zhang, Z., Fitzgerald, G. F. \& van Sinderen, D. (2005c). The clpB gene of Bifidobacterium breve UCC 2003: transcriptional analysis and first insights into stress induction. Microbiology 151, 2861-2872.

Ventura, M., Zhang, Z., Cronin, M., Canchaya, C., Kenny, J. G., Fitzgerald, G. F. \& van Sinderen, D. (2005d). The ClgR protein regulates transcription of the $c l p \mathrm{P}$ operon in Bifidobacterium breve UCC 2003. J Bacteriol 187, 8411-8426.

Ventura, M., Zink, R., Fitzgerald, G. F. \& van Sinderen, D. (2005e). Gene structure and transcriptional organization of the dnaK operon of Bifidobacterium breve UCC 2003 and application of the operon in bifidobacterial tracing. Appl Environ Microbiol 71, 487-500.

Ventura, M., Canchaya, C., Zhang, Z., Bernini, V., Fitzgerald, G. F. \& van Sinderen, D. (2006). How high G $+C$ Gram-positive bacteria and in particular bifidobacteria cope with heat stress: protein players and regulators. FEMS Microbiol Rev 30, 734-759.

WHO (2002). Active ageing: a policy framework. A contribution of the World Health Organization to the Second United Nations world assembly on ageing, Madrid, Spain, April 2002. http://whqlibdoc. who.int/hq/2002/WHO_NMH_NPH_02.8.pdf

Edited by: M. Kleerebezem 